PROCEEDINGS OF THE

AMERICAN MATHEMATICAL SOCIETY

Volume 125, Number 3, March 1997, Pages 835-837

S 0002-9939(97)03706-4

\title{
NOT ALL JULIA SETS ARE QUASI-SELF-SIMILAR
}

\author{
PENTTI JÄRVI
}

(Communicated by Albert Baernstein II)

\begin{abstract}
We show that there exist rational functions, whose Julia set fails
\end{abstract} to be quasi-self-similar.

1.

One of the conspicuous features of the Julia sets of rational functions is that small parts of them look very much like some large parts. Sullivan has introduced a proper concept to describe the situation: the quasi-self-similarity. He also established the quasi-self-similarity of the Julia sets of all hyperbolic rational functions $[1$, Theorem 8.6], [3, Theorem 7], [8, p. 742]. One of the open problems listed at the end of [3] asks, whether the same is true of all rational functions of degree $\geq 2$. The purpose of the present note is to show that this is not the case.

2.

Let $c \in(0,1]$. A set $E$ in the euclidean $n$-space $\mathbb{R}^{n}$ is $c$-porous if each closed ball $\bar{B}^{n}(x, r) \subset \mathbb{R}^{n}$ contains a point $z$ such that the open ball $B^{n}(z, c r)$ does not meet $E ; E$ is porous if it is $c$-porous for some $c$ (see e.g. [10]). For instance, Cantor sets with constant ratio in $\mathbb{R}^{n}$ are porous in $\mathbb{R}^{n}$. Given $k>0$, we let $\phi_{k}$ stand for the similarity map $x \mapsto k x, x \in \mathbb{R}^{n}$. A nonempty set $E \subset \mathbb{R}^{n}$ is called $K$ quasi-self-similar if there is an $r_{0}>0$ such that, given any closed ball $\bar{B}^{n}(x, r)$ with $t=r_{0} / r>1$, there exists a $K$-quasi-isometry $f: \phi_{t}\left(\bar{B}^{n}(x, r) \cap E\right) \rightarrow E$, i.e., $f$ satisfies

$$
\frac{1}{K}|y-z| \leq|f(y)-f(z)| \leq K|y-z| \quad \text { for all } y, z \in \phi_{t}\left(\bar{B}^{n}(x, r) \cap E\right) .
$$

Quasi-self-similarity means $K$-quasi-self-similarity for some $K \geq 1$. See [1, p. 121], [3, p. 65], [4, p. 183]. The constant $r_{0}$ is called a standard size of $E$ [4, p. 183]. We are going to show that quasi-self-similarity implies porosity under some mild restrictions.

Lemma. Let $E \subset \mathbb{R}^{n}$ be a compact, nowhere dense, quasi-self-similar set. Then $E$ is porous in $\mathbb{R}^{n}$.

Received by the editors September 19, 1995.

1991 Mathematics Subject Classification. Primary 30D05, 58F08.

Key words and phrases. Iteration, rational function, Julia set, quasi-self-similar set, porous set.

(C) 1997 American Mathematical Society 
Proof. Let $K$ be a constant of quasi-self-similarity of $E$, and let $r_{0}$ be a standard size of $E$. Assume that $E$ fails to be porous. Then we find a sequence $\left(x_{j}\right)$ of points in $\mathbb{R}^{n}$ and a sequence $\left(r_{j}\right)$ of radii such that if $B^{n}\left(z_{j}, r_{j}^{\prime}\right)$ is the maximal open ball with $z_{j} \in \bar{B}^{n}\left(x_{j}, r_{j}\right)$ and $B^{n}\left(z_{j}, r_{j}^{\prime}\right) \cap E=\varnothing$, then $r_{j}^{\prime} / r_{j}$ converges to 0 . Another way to express this state of affairs is to say that $A_{j}=\phi_{t_{j}}\left(\bar{B}^{n}\left(x_{j}, r_{j}\right) \cap E\right)-\phi_{t_{j}}\left(x_{j}\right)$ converges to $\bar{B}^{n}\left(0, r_{0}\right)=\bar{B}^{n}\left(r_{0}\right)$ in the Hausdorff metric, defined in the space of nonempty compact subsets of $\mathbb{R}^{n}$. We have used the standard notation $A-y=\{x-y \mid x \in A\}$ and the abbreviation $t_{j}=r_{0} / r_{j}$. We will see that this convergence property permits us to construct a $K$-quasi-isometry from $\bar{B}^{n}\left(r_{0}\right)$ into $E$.

Obviously $r_{j}$ tends to 0 as $j \rightarrow \infty$. Hence we may assume that $r_{j}<r_{0}$ for all $j \geq 1$. By assumption, there exists a $K$-quasi-isometry $f_{j}: A_{j} \rightarrow E$ for each $j \geq 1$. Let $C=\left\{y_{i} \mid i \in N\right\}$ be a countable dense subset of $\bar{B}^{n}\left(r_{0}\right)$. Since $A_{j} \rightarrow \bar{B}^{n}\left(r_{0}\right)$ in the Hausdorff metric, we find for each $i \in N$ a sequence $\left(y_{i j}\right)$ such that $y_{i j} \in A_{j}$ for all $i, j$ and $\lim _{j \rightarrow \infty} y_{i j}=y_{i}$. Since $E$ is compact, there is a subsequence $\left(y_{1 j_{1 k}}\right)$ of $\left(y_{1 j}\right)$ such that $\lim _{k \rightarrow \infty} f_{j_{1 k}}\left(y_{1 j_{1 k}}\right)$ exists. Similarly, we can find a subsequence $\left(j_{2 k}\right)$ of $\left(j_{1 k}\right)$ such that $\lim _{k \rightarrow \infty} f_{j_{2 k}}\left(y_{2 j_{2 k}}\right)$ exists. We proceed in this manner. Finally, thanks to the well-known diagonal process, we find a strictly increasing sequence of indices, say $\left(j_{k}\right)$, such that $\lim _{k \rightarrow \infty} f_{j_{k}}\left(y_{i j_{k}}\right)$ exists for each $i \in N$. Hence we are in a position to define

$$
f: C \rightarrow \mathbb{R}^{n}, \quad f\left(y_{i}\right)=\lim _{k \rightarrow \infty} f_{j_{k}}\left(y_{i j_{k}}\right) .
$$

Obviously $f(C) \subset E$.

We next verify that $f$ is a $K$-quasi-isometry. Let $x, y \in C$, say $x=y_{h}, y=y_{i}$, and let $\varepsilon>0$. Pick $k \in N$ such that $\left|y_{h}-y_{h j_{k}}\right|<\varepsilon,\left|y_{i}-y_{i j_{k}}\right|<\varepsilon,\left|f\left(y_{h}\right)-f_{j_{k}}\left(y_{h j_{k}}\right)\right|<\varepsilon$ and $\left|f\left(y_{i}\right)-f_{j_{k}}\left(y_{i j_{k}}\right)\right|<\varepsilon$. Since $f_{j_{k}}: A_{j_{k}} \rightarrow E$ is a $K$-quasi-isometry, we have

$$
\left|f_{j_{k}}\left(y_{h j_{k}}\right)-f_{j_{k}}\left(y_{i j_{k}}\right)\right| \leq K\left|y_{h j_{k}}-y_{i j_{k}}\right| \text {. }
$$

Hence

$$
\begin{aligned}
|f(x)-f(y)| & =\left|f\left(y_{h}\right)-f\left(y_{i}\right)\right|<2 \varepsilon+K\left|y_{h j_{k}}-y_{i j_{k}}\right| \\
& <2 \varepsilon+K\left(2 \varepsilon+\left|y_{h}-y_{i}\right|\right)=2(1+K) \varepsilon+K|x-y| .
\end{aligned}
$$

Letting $\varepsilon \rightarrow 0$ gives the right-hand side of (1). The proof of the left-hand inequality is similar.

Now $f$ is uniformly continuous in a dense subset of $\bar{B}^{n}\left(r_{0}\right)$. This implies that $f$ admits a continuous extension $f^{*}: \bar{B}^{n}\left(r_{0}\right) \rightarrow E$. It is again a simple matter to verify that $f^{*}$ is a $K$-quasi-isometry. But this contradicts the assumption that $E$ is nowhere dense. The proof is complete.

3.

It is not too difficult to show, making use of net measures, that the Hausdorff dimension of every set, which is porous in $\mathbb{R}^{n}$, is less than $n$. See e.g. $[9$, p. 127]. Hence we deduce

Corollary 1. Let $E \subset \mathbb{R}^{n}$ be a compact, nowhere dense, quasi-self-similar set. Then $\operatorname{dim}_{H}(E)$, the Hausdorff dimension of $E$, is less than $n$.

Consider now the family of complex quadratic polynomials

$$
f_{c}(z)=z^{2}+c, \quad c \in \mathbb{C},
$$


and let $J\left(f_{c}\right)$ denote the Julia set of $f_{c}$. Note that $J\left(f_{c}\right)$ is a compact nowhere dense subset of $\mathbb{C}$, because $\infty$ belongs to the Fatou set of $f_{c}$. Shishikura [5], [6] has recently shown that there are values of $c$ for which $\operatorname{dim}_{H}\left(J\left(f_{c}\right)\right)=2$. More precisely, there is a residual subset $F$ of the boundary of the Mandelbrot set such that if $c \in F$, then $\operatorname{dim}_{H}\left(J\left(f_{c}\right)\right)=2$. Hence we have

Corollary 2. There are values $c \in \mathbb{C}$ such that $J\left(f_{c}\right)$ fails to be quasi-self-similar.

Remark 1. As mentioned above, the Julia set of any hyperbolic rational function is quasi-self-similar (see [2, pp. 89-93] for basic properties of hyperbolic rational maps). It follows from Corollary 1 that $\operatorname{dim}_{H}(J(f))<2$ for such functions. Sullivan $[8$, Theorem 4] has deduced this result relying on properties of conformal measures defined on Julia sets.

Remark 2. Of course, the same question can be proposed in the context of finitely generated Kleinian groups; that is, is the limit set of any finitely generated Kleinian group of $\overline{\mathbb{R}}^{n}$ quasi-self-similar? The answer is again in the negative. This follows, in view of Corollary 1, from a result of Sullivan [7], according to which there are finitely generated Kleinian groups of $\overline{\mathbb{R}}^{2}$ whose limit set has Hausdorff dimension two. Note, however, that the Hausdorff dimension of a geometrically finite Kleinian group of $\overline{\mathbb{R}}^{n}$ is always less than $n[9$, Theorem D].

\section{REFERENCES}

1. P. Blanchard, Complex analytic dynamics on the Riemann sphere, Bull. Amer. Math. Soc. 11 (1984), 85-141. MR 85h:58001

2. L. Carleson and T. W. Gamelin, Complex Dynamics, Springer-Verlag, Berlin, 1993. MR 94h:30033

3. L. Keen, Julia sets, Chaos and Fractals: The Mathematics Behind the Computer Graphics (L. Keen and R. Devaney, eds.), Amer. Math. Soc., Providence, 1989, pp. 57-74. MR 91a:58130

4. J. McLaughlin, A note on Hausdorff measures of quasi-self-similar sets, Proc. Amer. Math. Soc. 100 (1987), 183-186. MR 88d:54054

5. M. Shishikura, The Hausdorff Dimension of the Boundary of the Mandelbrot Set and Julia Sets, Preprint 1991/7, SUNY Stony Brook, IMS.

6. M. Shishikura, The boundary of the Mandelbrot set has Hausdorff dimension two, Astérisque 222 (1994), 389-405. CMP 94:15

7. D. Sullivan, Growth of positive harmonic functions and Kleinian group limit sets of zero planar measure and Hausdorff dimension two, Geometry Symposium Utrecht 1980 (E. Looijenga, D. Siersma, and F. Takens, eds.), Lecture Notes in Math., vol. 894, Springer-Verlag, Berlin, 1981, pp. 127-144. MR 83h:53054

8. D. Sullivan, Conformal dynamical systems, Geometric Dynamics (J. Palis, ed.), Lecture Notes in Math., vol. 1007, Springer-Verlag, Berlin, 1983, pp. 725-752. MR 85m:58112

9. P. Tukia, The Hausdorff dimension of the limit set of a geometrically finite Kleinian group, Acta Math. 152 (1984), 127-140. MR 85m:30031

10. J. Väisälä, Porous sets and quasisymmetric maps, Trans. Amer. Math. Soc. 299 (1987), 525533. MR 88a:30049

Department of Mathematics, University of Helsinki, P.O. Box 4 (Hallituskatu 15), FIN-00014 Helsinki, FinLAND 